Conf-920362--20

GA-A- -20860

DE92 011394

\title{
REAL TIME ANALYSIS OF TOKAMAK DISCHARGE PARAMETERS
}

\author{
by \\ J.R. FERRON and E.J. STRAIT
}

MARCH 1992

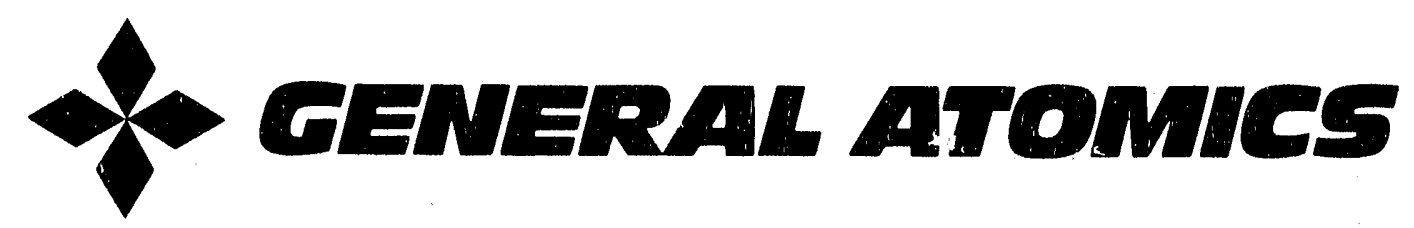




\section{DISCLAIMER}

'This report was prepared as an account of work sponsored by an agency of the United States Government. Neither the United States Government nor any agency thereof, nor any of their employees, makes any warranty, express or implied, or assumea any legal liability or responuibility for the accirracy, completeness, or usefulness of any informaiviva, apparatus, product, or process disclosed, or represents that its use would not infringe privately owned rights. Reference herein to any specific commercial product, process, or service by trade name, trademark, manufacturer, or otherwise, does not necesserily constitute or imply its endorsement, recummendation, of favoring by the United States Government or any agency thereof. The views and opinions of authors expressed herein do not necessarily state or reflect those of the United States Government or any agency thereof. 
GA-A20860

\title{
REAL TIME ANALYSIS OF TOKAMAK DISCHARGE PARAMETERS
}

\author{
by \\ J.R. FERRON and E.J. STRAIT
}

This is a preprint of a paper presented at the 9th Topical Conference on High-Temperature Plasma Diagnostics, March 16-20, 1992, Santa Fe, I ew Mexico, and to be printed in Review of Scientific Instruments.

Work supported by

U.S. Department of Energy

Contract No. DE-AC03-89ER51114

\author{
GENERAL ATOMICS PROJECT 3466 \\ MARCH 1992
}

\section{GENERAL ATOMICS}




\title{
Real time analysis of tokamak discharge parameters
}

\author{
J.R. FERron and E.J. Strait
}

General Atomics, San Diego, California 92186-9784

The techniques used in implementing two applications of real time analysis of data from the DIII-D tokamak are described. These tasks, which are demanding in both the speed of data acquisition and the speed of computation, execute on hardware capable of acquiring 40 million data samples per second and executing 80 million floating point operations per second. In the first case, a feedback control algorithm executing at a $10 \mathrm{kHz}$ cycle frequency is used to specify the current in the poloidal field coils in order to control the discharge shape. In the second, fast Fourier transforms of Mirnov probe data are used to find the amplitude and frequency of each of eight toroidal mode numbers as a function of time during the discharge. Data sampled continuously at $500 \mathrm{kHz}$ are used to produce results at $2 \mathrm{msec}$ intervals. 


\section{INTRODUCTION}

Real time digital analysis of tokamak data is becoming necessary for the more complex tasks in present-day facilities and will be indispensible for the the next generation of long pulse or steady state tokamaks. Here, real time analysis refers to data processing which is performed on the time scale of acquisition of the data while the tokamak discharge is operating. In long pulse or steady state discharges, the quantity of continuously sampled diagnostic data could easily become unmanageable. Real time analysis can be used for reduction of data to fewer values for storage, generation of trigger signals to indicate time periods for which unprocessed diagnostic data should be saved, or to generate real time data displays for experimenters. The detailed control of tokamak discharges envisioned for the next generation devices will require real time analysis in order to evaluate the complex quantities to be controlled such as the current and pressure profiles. Real time diagnostic analysis would be required to provide processed results to the discharge control systems.

To make real time analysis possible both a high speed digital processor and a high rate of transfer of data between the diagnostic detector and the digital processor are necessary. A high speed data acquisition and processing system which satisfies these requirements has been developed for use in plasma control and diagnostic data analysis applications for the DIII-D tokamak. ${ }^{1-2}$ In this paper we describe the implementation of two applications with this system: control of the shape of the tokamak discharge and spectral analysis of fluctuations in the magnetic field detected by Mirnov probes. The type of real time calculation described here has not been practical previously but can now be implemented because of recent improvements in the processing speed of readily available microprocessors. In the remainder of this paper, we give a brief description of the hardware in use for real time data analysis (Section I), followed by a description of the two real time calculations in Sections II and III. 


\section{THE DATA ACQUISITION AND PROCESSING HARDWARE}

The hardware for the general purpose data acquisition and processing system used in this work has been described in detail in Ref. 2 so only a brief description is given here. The digital processor is an Intel i860 microprocessor with a clock speed of $40 \mathrm{MHz}$ and peak processing speeds of $40 \times 10^{6}$ integer instructions per second simultaneously with $80 \times 10^{6}$ floating point operations per second. There are 16 Mbytes of high speed random access memory which can be used for data and software storage. Data from the tokamak diagnostic is transfered into a firstin, first-out (FIFO) memory where it is buffered until the i 860 processor requests data. The data is then transferred directly from the FIFO memory into the random access memory without further intervention by the i860 processor. Buffering of the data in a FIFO memory allows data to be delivered to multiple processor systems simultaneously for parallel processing applications without requiring any synchronization between the processors. Data can be transferred from the diagnostic to the FIFO memory and from there to the random access memory at a peak speed of $40 \times 10^{6}$, fourteen bit samples per second. There is also an interface to the industry standard VME bus which allows access to a wide variety of input and output devices which might be necessary in a specific application and to a Sun Microsystems computer which acts as the host computer and provides the user interface.

The data acquisition hardware is designed to accommodate two primary applications: feedback control and data reduction. In feedback control a single set of diagnostic data samples is collected and the commands for the tokamak systems are calculated and output to the tokamak in each feedback cycle. The calculation cycle period and the time delay between the sampling of the diagnostic signals and the output of the command must be kept to a minimum. Therefore the data is transferred from the diagnostic to the FIFO memory simultaneously with the transfer from the FIFO memory to the random access memory. In data reduction the processor is only 
required to have an average processing rate which is as fast as the average rate at which data is acquired. The data is typically buffered in the FIFO memory until a sufficient number of samples has accumulated and then it is written to the random access memory in a relatively long burst. The data are accumulated in one buffer in the random access memory while the processor reduces the data stored in a second memory buffer.

The processor cannot access the memory while the data transfer from the FIFO memory is in progress, so the amount of time available for memory access during data processing depends on how close the average rate of data transfer is to the maximum value and the extent to which the processor's cache memory can be used during the analysis. If there is not sufficient time available for analysis the entire random access memory can be used to store the data acquired from the diagnostic during the tokamak discharge. The i860 processor can then provide reduced data in "near real time," that is, on a short time scale after the discharge ends.

\section{A FEEDBACK CONTROL APPLICATION}

In this section, we discuss the techniques used to achieve a $10 \mathrm{kHz}$ feedback control cycle frequency with the computation intensive algorithm used for control of the DIII-D tokamak discharge shape. The DIII-D tokamak discharge shape is regulated by controlling the current in the 18 poloidal field coils. The current in each coil is controlled by a separate power supply so 18 power supply commands must be generated in each feedback system computation cycle. 96 tokamak magnetic diagnostic signals are digitized to provide the input data. The control algorithm uses a predetermined linear mapping, implemented with a matrix multiplication, between the input data and the discharge shape to determine the difference between the actual shape and the desired shape. ${ }^{1}$ On each cycle of the control system the 96 input values are used to compute the errors in the 18 aspects of the discharge shape that are to be controlled and the values of beta poloidal and the internal inductance which are 
used to choose the appropriate linear mapping. The errors are then used to compute the commands for the poloidal field power supplies.

During a discharge, the control system loops continuously through its processing cycle. First, at the appropriate time in the previous cycle, the i860 processor sends a signal to the digitizers to trigger conversion of the 96 analog input signals to digital values. The analog to digital conversion requires a total of $7 \mu \mathrm{sec}$ and an additional $3.2 \mu \mathrm{sec}$ are required to transfer the data from the digitizers to the random access memory. This is accomplished while the processor completes a control cycle using the previous set of data. The data are then converted by the i860 from integer to floating point format ${ }^{2}$ in a total of $2.4 \mu \mathrm{sec}$.

Second, the linear mapping is used to compute the 18 discharge shape parameters to be controlled plus $\beta_{p}$ and $\ell_{i}$. This requires multiplication of the 96 element data vector by a $96 \times 20$ control matrix. The operation involves 3840 floating point multiplications or additions and requires only $49.7 \mu \mathrm{sec}$. The computed shape parameter values are subtracted from the desired values to obtain the error in the shape.

Finally, the 18 element error vector is translated to commands to increase or decrease the current in the poloidal field coils through multiplication by an $18 \times 18$ matrix that specifies which coil currents should be changed in order to adjust each of the shape parameters. This calculation requires $12.7 \mu \mathrm{sec}$. The coil current commands are delivered to the poloidal field power supplies by writing the command levels to digital to analog converters using the VME bus.

The total cycle time is less than $100 \mu \mathrm{sec}$ although a large amount of floating point computation is required. This is achieved by taking advantage of several features of the hardware system.

1. The data is collected from the digitizers in a short burst at the peak speed of 40 Msamples/sec so there is a very short delay between sampling the analog signal and the beginning of the computation. 
2. The $i 860$ processor is specifically designed for vector operations so calculation rates within a few percent of the theoretical maximum of 80 MFLOPS can actually be sustained during the matrix operations which take most of the time in the algorithm.

3. Access to the on-chip cache memory of the $i 860$ processor can be sustained at $640 \mathrm{Mbyte} / \mathrm{sec}$. Therefore, the software is arranged so that most of the smaller vectors that hold intermediate results are stored in the cache memory. The data cache of 8 kbyte is too small to hold the rather large control matrix along with everything else so the $\mathrm{i} 860$ "pipelined floating load" instruction is used to read the this matrix at maximum speed without disturbing the cache content.

4. The entire content of the control matrix can be moved into the processor from memory during the matrix multiplication without reducing the floating point calculation rate because the processor memory interface provides for $160 \mathrm{Mbyte} / \mathrm{sec}$ peak transfer speeds and rates within a few percent of this value are possible during the matrix multiplication.

5. The entire program is composed of fewer than the 1000 machine language instructions which can be stored in the instruction cache. Because the program repeatedly executes the same instructions, access to the random access memory is not required to fetch instructions so the entire bandwidth of the processormemory interface is available for the transfer of data for the calculations.

6. During the matrix multiplication which produces the control command values, as the multiplication of the error vector by each matrix row is completed, the value is written immediately to the digital to analog convertor. The processor can perform the required memory bus operations simultaneously with the continuation of the matrix multiplication because all components of this multiplication are located in the data cache. 


\section{MODE NUMBER ANALYSIS OF MIRNOV FLUCTUATIONS}

One of the more useful tokamak diagnostics is an array of Mirnov probes which measure fluctuations in the magnetic field just outside the plasma. Spectral analysis ${ }^{3}$ of these fluctuations is a powerful tool for the detection of plasma instabilities which may be present in the discharge. Continusus acquisition of Mirnov probe data at a sufficiently high sampling rate is typically not possible because of constraints on available digitizer memory. In this section, we describe how real time analysis of the fluctuation data from a pair of Mirnov probes has been implemented to allow observation of spectra throughout a discharge.

Frequency, amplitude, and mode number are obtained from the smoothed cross power spectrum of the Mirnov probe pair. With $X_{1}(\omega)$ and $X_{2}(\omega)$ the complex Fourier transforms of the digitized magnetic fluctuation signals from the probes, the complex cross-spectrum of the probe pair is $C_{12}(\omega)=\left\langle X_{1}(\omega) X_{2}^{*}(\omega)\right\rangle$, where " \langle\rangle $"$ denotes averaging in the frequency domain over an interval $\delta \omega$, centered about $\omega$. From $C_{12}(\omega)$ we derive the cross-power, phase, and coherence. The cross-power spectrum $P_{12}(\omega)=\left(C_{12}(\omega) C_{12}^{*}(\omega)\right)^{1 / 2}$ represents the fluctuation power density versus frequiency. The phase spectrum $\Theta_{12}(\omega)=\tan ^{-1}\left(\operatorname{Im}\left[C_{12}(\omega)\right] / \operatorname{Re}\left[C_{12}(\omega)\right]\right)$ gives the phase difference between the probes versus frequency. The toroidal mode number versus frequency can be estimated by $n(\omega)=\Theta_{12}(\omega) / \Delta \Phi$ rounded to the nearest integer, where $\Delta \Phi$ is the toroidal angle separating the probes. The coherence spectrum for the probe pair is defined as $\gamma_{12}(\omega)=P_{12}(\omega) /\left[P_{11}(\omega) P_{22}(\omega)\right]^{1 / 2}$, where $P_{11}$ and $P_{22}$ are the analogous auto-power spectra of the individual probes. A high value of $\gamma_{12}(\omega)$ indicates that the two probe signals are well-correlated at the frequency $\omega$, while a low value suggests that the probes are measuring uncorrelated noise. The $95 \%$ confidence level for correlation is approximately given by $\gamma_{95 \%}=\tanh (1.96 / \sqrt{2 M-2})$, where $M$ is the number of frequency elements included in the averaging interval $\delta \omega$. 
This provides a convenient criterion for deciding whether a peak in the cross-power spectrum is meaningful, and whether the associated mode number estimate is reliable.

The input data for the calculation is a set of 1024 samples from each of two probes separated by $45^{\circ}$ toroidally from which eight toroidal mode numbers can be distinguished. The real time algorithm calculates the mode number versus frequency from each set of data and records the peak amplitude and the frequency at the peak for each of the eight detectable mode numbers, ignoring frequencies at which $\gamma_{12}<\gamma_{95 \%}$. Typically the lower and higher frequency ranges represent different instabilities, so the amplitude peaks are recorded in two frequency ranges. Thus the data quantity is reduced by a factor of 32 from the 1024 twelve bit integer samples from each of the two probes, requiring a total of 4096 bytes of storage, to 16 floating point values in each of two frequency ranges, requiring 128 bytes of storage.

Each set of data is acquired by using the FIFO memory as a buffer. The $i 860$ processor programs the "data transfer counter," part of the data acquisition hardware, with the number of samples required from each diagnostic channel. The sample trigger for the digitizers is generated by the tokamak timing system at $2 \mu$ sec intervals beginning at some specified tirse during the discharge. After each sample trigger is received, digitizer data is transferred into the FIFO memory. When the FIFO memory becomes half full, a transfer of data into the random access memory is triggered. This transfer lasts until the FIFO memory is empty. Each time the FIFO memory becomes half full there is a new data transfer triggered until the specified total number of samples has been acquired. Because there are only two digitizer channels written into the FIFO every $2 \mu \mathrm{sec}$, the input rate is $1 \mathrm{Msample} / \mathrm{sec}$, whereas the data is written to the random access memory at $10 \mathrm{Msamples} / \mathrm{sec}$. Thus, during only $55 \mu \mathrm{sec}$ of the $2048 \mu \mathrm{sec}$ required to acquire the data, the random access memory is busy with the data transfer and during the remaining $1993 \mu \mathrm{sec}$ the $\mathrm{i} 860$ processor can access the memory during the data analysis. 
Second, the vectors $X_{1}(\omega)$ and $X_{2}(\omega)$ are passed through a pipelined calculation which is optimized for use with the floating point multiply and add pipeline in the i860 processor. The first part of the pipeline calculates the real and imaginary parts of $X_{1}(\omega) X_{2}^{*}(\omega)$, and the real values $X_{1}(\omega) X_{1}^{*}(\omega)$ and $X_{2}(\omega) X_{2}^{*}(\omega)$. In the second part of the pipeline these four values are separately averaged over three points in the frequency domain. To compute one averaged vector element requires two multiplies and three additions which are performed in 4 processor clock cycles. The final part of the pipeline computes $P_{11}(\omega) P_{22}(\omega) \gamma_{\theta 5 \%}^{2}$ and $P_{12}^{2}$ in five clocks. Thus 21 clocks per vector element are required for a total of $27 \hat{\theta} \mu$ sec for 512 vector elements.

The complex vector $C_{12}$ and the two real vectors $P_{12}^{2}$ and $P_{11}(\omega) P_{22}(\omega) \gamma_{95 \%}^{2}$ contain all of the information necessary to find the frequency peaks and assoriated mode numbers. The actual calculation of the phase is not necessary. Instead, the mode number at each frequency is found by comparing $\operatorname{Im}\left[C_{12}(\omega)\right]$ to each value of $\tan \left(\theta_{i}\right) \operatorname{Re}\left[C_{12}(\omega)\right]$ where $\tan \left(\theta_{i}\right)$ is an entry from a table of values of the tangents of the angles which separate the complex plane into eight regions, one for each mode number. Each vector element is tested for sufficient coherence by comparing $P_{12}^{2}$ to $P_{12}(\omega) P_{11}(\omega) \gamma_{95 \%}^{2}$. Finally, the maximum amplitude and its frequency are recorded for each mode number using only frequency points with sufficient coherence. The calculation of the mode number and the peak amplitudes requires multiplications by the $\tan \left(\theta_{i}\right)$ values and several comparison operations, using a maximum of 31 processor clocks per point or $400 \mu \mathrm{sec}$.

The complete calculation, then, requires approximately $1915 \mu \mathrm{sec}$, less than the time required to acquire the data. This very fast spectral analysis is possible because of the vector processing features in the i860 processor, because assembly language code was written to optimize the implementation of the algorithm and because unnecessary calculation intensive operations such as divisions, inverse tangent and square roots were eliminated from the algorithm. 


\section{SUMMARY}

We have described how two data acquisition and computation intensive applications can be implemented in real time using digital analysis hardware which can both acquire data and perform floating point computation at high speed. These are only two examples of the type of real time data analysis application which can be useful in present tokamak facilities and which will be essential in future long pulse tokamaks.

\section{ACKNOWLEDCMENT}

This work was supported by the U.S. Department of Energy under Contract No. DE-AC03-89ER51114. 


\section{REFERENCES}

1 J. R. Ferron, etal., "An Advanced Plasma Control System for the DIII-D Tokamak," Proceedings of the 14th IEEE/NPS Symposium on Fusion Engineering (IEEE, to be published), 1991.

2 J. R. Ferron, "A High Speed Data Acquisition and Processinig System for Real Time Data Analysis and Control", General Atomics Report GA-A20810, submitted for publication in Rev. Sci. Instru.

3 G. M. Jenkins and D. G. Watts, Spectral Analysis, Holden-Day, San Francisco, 1968. 

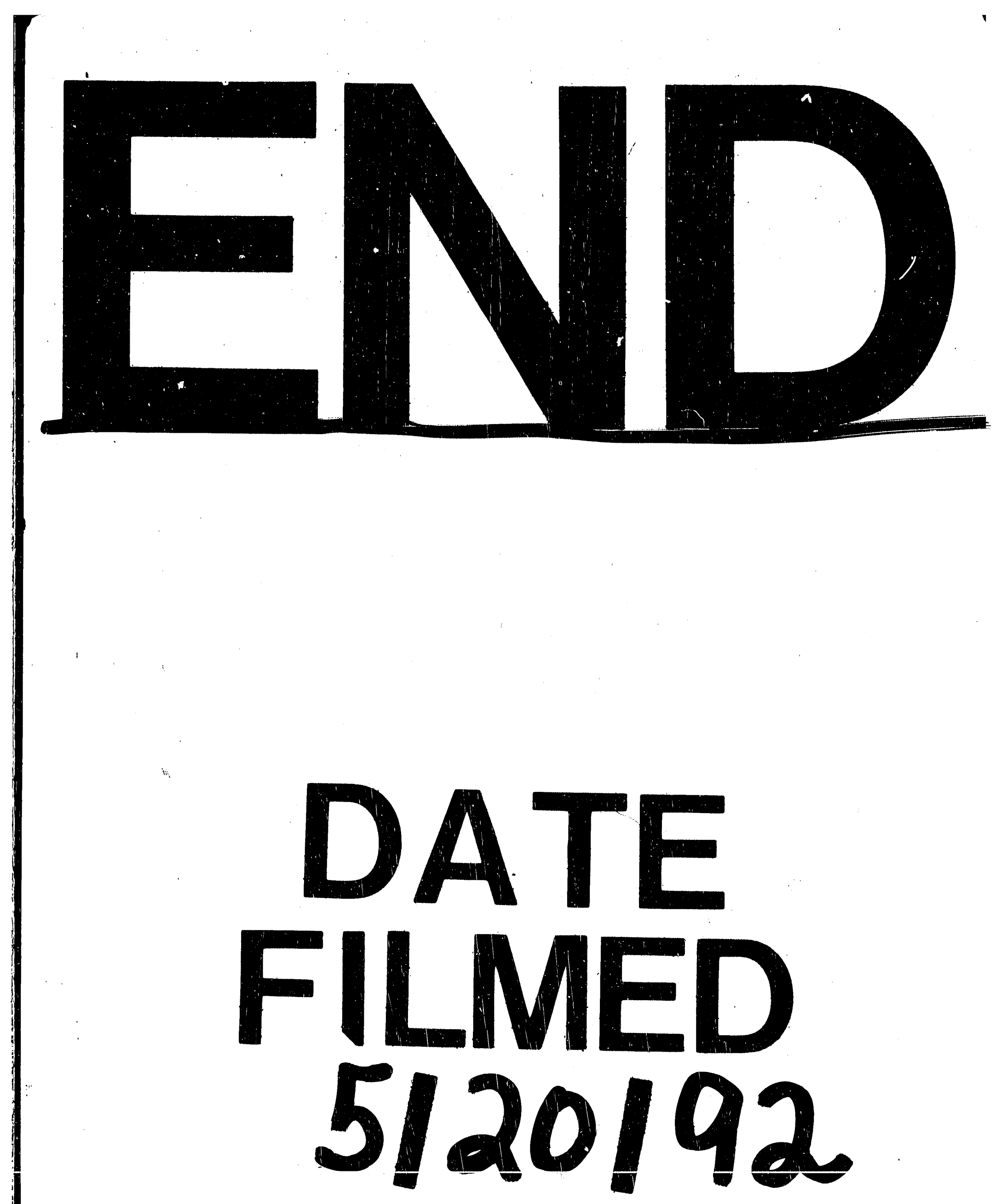


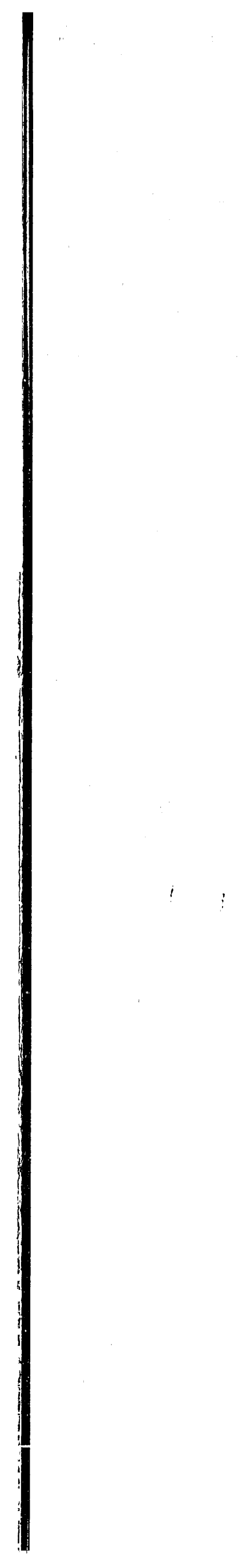

